\title{
Risk factors for peritonitis related to peritoneal dialysis
}

\author{
Karagulle IV ${ }^{1}$, Kaynar $\mathrm{K}^{2}$, Ulusoy $\mathrm{S}^{2}$, Ozkan $\mathrm{G}^{2}$, Cansiz $\mathrm{M}^{2}$ \\ Department of Internal Medicine, School of Medicine, Karadeniz Technical University, Trabzon, Turkey.
} kkaynar@yahoo.com

\begin{abstract}
Background: The aim of this study was to evaluate risk factors for peritonitis associated with peritoneal dialysis in our centre.

Methods: Forty patients on chronic peritoneal dialysis between 2006-2010 were enrolled in this study. Nutritional, sociodemographical, psychological, dialysis related (peritoneal modality, characteristics and adequacy) possible risk factors associated with the development of peritonitis were recorded. Data of patients with and without peritonitis were compared using the Student's t-test and chi-square test.

Results: There were 31 episodes of peritonitis among 21 patients, while other 19 patients had not experienced peritonitis resulting in a rate of 1 episode / 44 patient months. Staphylococcus aureus was the most frequent microorganism isolated (31\%). Hypoalbuminemia, technical problems, poor personal and environmental hygiene, low educational status, current or former smoking history and less urine output were observed significantly more in patients who had peritonitis.

Conclusions: Our results confirm that educational, psychological, nutritional, smoking and hygiene status and amount of urine output are closely associated with peritonitis among peritoneal dialysis patients. Measures to improve these factors may help prevent and control peritoneal dialysis related peritonitis (Tab. 4, Ref. 14). Full Text in PDF www.elis.sk.

Key words: peritoneal dialysis, peritonitis, low education, hypoalbuminemia, poor hygiene status, low urine output.
\end{abstract}

Even though the rate of chronic peritoneal dialysis (CPD) associated peritonitis has decreased since CPD was first described, peritonitis is still a frequent complication of peritoneal dialysis and the most common cause of increased morbidity and mortality in patients with peritoneal dialysis (1). Incidence and risk factors of peritonitis associated with peritoneal dialysis (PD) differ between countries and even between clinics in the same country. The aim of this study was to evaluate risk factors for peritonitis associated with peritoneal dialysis in our centre.

\section{Material and methods}

Between 2006-2010, totally 40 patients were studied retrospectively in the Renal outpatient clinic of Karadeniz Technical University, School of Medicine. This study was approved by the Karadeniz Technical University Ethics Committee. The following basic parameters; aetiology of end stage renal disease, type of $\mathrm{PD}$, gender, occupation, education level (university, high school or lower), weight, height, body mass index (calculated as weight - kilograms - divided by height - meters - squared), psychosocial

${ }^{1}$ Department of Internal Medicine, School of Medicine, Karadeniz Technical University, Trabzon, Turkey, and ${ }^{2}$ Department of Nephrology, School of Medicine, Karadeniz Technical University, Trabzon, Turkey

Address for correspondence: K. Kaynar, MD, Karadeniz Teknik Üniversitesi, Tip Fakültesi Nefroloji Bilim Dali, 61080 Trabzon, Turkey. Phone: +90.542 .2415879 , Fax: +90.462.3252270

Acknowledgements: We thank Professor Nuray Misir for her skilful assistance in statistical analysis. status, smoking habits, immunosuppressive drug intake, usage of antibiotics and proton pump inhibitors, history of intra-abdominal operation and endoscopic procedure, constipation, presence of personal hygiene deficits (like self hygiene neglect, making dialysis exchanges in inappropriate environment), presence of environmental hygiene deficits (like living in rural areas, living with infected people), presence of technical problems (like leakage around the catheter, perforation in dialysis bags, technical problems developed during catheter insertion, usage of nonsterile caps), nasal staphylococcus aureus carriage, presence of residual renal functions, peritoneal transport characteristics, presence of comorbid disease (myocardial infarction, heart failure, peripheral vascular disease, dementia, chronic pulmonary disease, connective tissue disease, duodenal ulcer, liver disease, diabetes mellitus, hemiplegia, leukemia, lymphoma, solid tumour, AIDS) as Charlson comorbidity index of patients were recorded (2).

All patients had a straight double-cuff Tenckhoff catheter inserted by open surgery and placed by experienced surgeons. Prophylactic ceftriaxone was administered before catheter placement in all cases. Mupirocin cream was recommended to be applied to the skin around the exit site after daily cleansing with antiseptics. All patients used the CAPD twin-bag system manufactured by Baxter Healthcare Corporation and Fresenius Medical Care. First renal replacement treatment modality, timing of transfer set exchange, place and date of peritoneal catheter insertion were noted. Clinical status of all patients was assessed in the outpatient clinics every month. Routine laboratory measurements including hemoglobin, total leukocyte count, blood urea nitrogen, albumin, calcium, phosphate, sodium, potassium, $\mathrm{C}$ reactive protein, ferritin and peritoneal 
$531-533$

fluid carcinoembrionic antigen (CEA) levels were documented during time of maintenance PD and peritonitis. Dialysis adequacy was assessed every 4 months via calculations of urea clearance $\mathrm{Kt} / \mathrm{V}$ from 24 hour urine and dialysate (3). The normalized protein catabolic rate (nPCR) was estimated by urea nitrogen generation rate normalized to body weight (4). Solute transport by peritoneum was evaluated by the peritoneal equilibration test (PET) (5).

Peritonitis was diagnosed with cloudy dialysate and/or abdominal pain, with dialysate effluent showing white blood cells $>100 \mathrm{~mm}^{3}$, and more than $50 \%$ polymorphonuclear neutrophils, microorganism detection in peritoneal fluid cultures. Peritonitis records of patients (date, etiologic agent, antibiotic regimens, duration of hospitalization) were collected. Peritonitis rate was calculated per patient month. Exit-site infection (defined as purulent discharge with positive culture) was also recorded.

\section{Statistical analysis}

We performed the Student's t-test for comparison of continuous variables expressed as the mean \pm standard deviation (SD). The chi-square test was used to compare categorical data using a computer-assisted programme with SPSS for Windows 11.5 (SPSS Inc., Chicago, USA). A value of $\mathrm{p}$ less than 0.05 indicated significance.

\section{Results}

Among 40 patients, 19 of them had never peritonitis between 2006-2010. 31 episodes of peritonitis were identified in the 21 patients over 4 years as compared to no episodes in the 19 control patients. That incidence amounted to 1 episode of peritonitis per 44 patient-months in all of 40 CPD population, and 1 episode of peritonitis per 25 patient-months in the $21 \mathrm{CPD}$ patients in the peritonitis group. The causes of end stage renal disease of patients were shown in the Table 1, leading primary renal disease was hypertension in both groups (groups were not different in this respect). Table 2 summarises the characteristics of the two groups, highlighting the higher educational level of control group, more current smoking and smoking history; more psychosocial abnormality like depression, anxiety disorders, technical problems, personal hygiene deficits, environmental hygiene deficits in peritonitis group.

Manual PD techniques (continuous ambulatory peritoneal dialysis) were used in 95 and $76 \%$ of patients in control and

Tab. 1. Comparison of peritonitis and control groups with respect to aetiology of end stage renal disease.

\begin{tabular}{lcc}
\hline Aetiology & $\begin{array}{c}\text { Control group } \\
\text { Number of patients } \\
\text { (percentage) }\end{array}$ & $\begin{array}{c}\text { Peritonitis group } \\
\text { Number of patients } \\
\text { (percentage) }\end{array}$ \\
\hline Glomerulonephritis & $3(16 \%)$ & $5(24 \%)$ \\
Diabetes Mellitus & $1(5 \%)$ & $1(5 \%)$ \\
Hypertension & $8(42 \%)$ & $12(57 \%)$ \\
Polycystic kidney disease & $1(5 \%)$ & $0(0 \%)$ \\
Others & $6(32 \%)$ & $3(14 \%)$ \\
\hline
\end{tabular}

The groups were not different according to aetiology of underlying end stage renal disease $\mathrm{p}>0.05$.
Tab. 2. Characteristic features of the peritonitis and control groups.

\begin{tabular}{|c|c|c|}
\hline Variables & $\begin{array}{l}\text { Peritonitis } \\
\text { group }\end{array}$ & $\begin{array}{c}\text { Control } \\
\text { group }\end{array}$ \\
\hline Age (years) ${ }^{\mathrm{b}}$ & $41 \pm 16$ & $33 \pm 12$ \\
\hline Female, $\%$ b & 47 & 52 \\
\hline Charlson index $\geq 3$ points, $\%{ }^{b}$ & 33 & 42 \\
\hline $\mathrm{DM}, \%$ b & 5 & 5 \\
\hline University graduate, $\%{ }^{a}$ & 0 & 44 \\
\hline Illiterate, $\%{ }^{\mathrm{a}}$ & 16 & 0 \\
\hline Education time for PD, hours/year ${ }^{b}$ & $6 \pm 13.5$ & $4.6 \pm 11$ \\
\hline Absence of smoking history, $\%{ }^{a}$ & 48 & 90 \\
\hline Normal psychosocial status, $\% \mathrm{a}^{\mathrm{a}}$ & 76 & 100 \\
\hline Usage of proton pump inhibitors, $\%{ }^{b}$ & 33.5 & 21 \\
\hline History of abdominal surgery, $\%$ b & 24 & 5 \\
\hline Presence of personal hygiene deficits, $\% \mathrm{a}^{\mathrm{a}}$ & 58 & 0 \\
\hline Presence of environmental hygiene deficits, $\%{ }^{a}$ & 32 & 0 \\
\hline Presence of technical problems, $\%^{\mathrm{a}}$ & 10 & 0 \\
\hline
\end{tabular}

Mean \pm Standard deviations, or percentage are shown, ${ }^{a} \mathrm{p}<0.05,{ }^{\mathrm{b}} \mathrm{p}>0.05$, DM: diabetes mellitus, PD: peritoneal dialysis, CAPD: continuous ambulatory peritoneal dialysis.

Tab. 3. Comparison of groups according to peritoneal dialysis features.

\begin{tabular}{lcc}
\hline Variables & $\begin{array}{c}\text { Peritonitis } \\
\text { group }\end{array}$ & $\begin{array}{c}\text { Control } \\
\text { group }\end{array}$ \\
\hline Duration of peritoneal dialysis (months) ${ }^{\mathrm{b}}$ & $30.0 \pm 5.9$ & $33.0 \pm 6.3$ \\
Type of PD, CAPD $\%^{\mathrm{b}}$ & 76 & 95 \\
$\mathrm{Kt}^{\mathrm{b}} \mathrm{V}^{\mathrm{b}}$ & $2.56 \pm 0.65$ & $2.56 \pm 0.54$ \\
Protein catabolic rate, $(\mathrm{g} / \mathrm{kg} / \mathrm{day})^{\mathrm{b}}$ & $0.95 \pm 0.15$ & $1.13 \pm 0.39$ \\
Urine output $>500 \mathrm{~mL} / \mathrm{day}, \mathrm{o}^{\mathrm{a}}$ & 33 & 73 \\
Carriage of nasal Staphylococcus aureus, $\%{ }^{\mathrm{b}}$ & 0 & 0 \\
Tunnel infection, $\%{ }^{\mathrm{b}}$ & 0 & 0 \\
Regular transfer set exchange, $\%{ }^{\mathrm{b}}$ & 81 & 100 \\
\hline Mean \pm Standard deviations, or percentage are shown, ${ }^{\mathrm{a}} \mathrm{p}<0.05,{ }^{\mathrm{b}} \mathrm{p}>0.05$.
\end{tabular}

Tab. 4. Comparison of groups according to laboratory parameters.

\begin{tabular}{lcc}
\hline Laboratory parameters & Control group & Peritonitis group \\
\hline Hemoglobin (g/dL)b & $11.5 \pm 1.9$ & $10.4 \pm 1.9$ \\
White blood cells(/mL)a & $7800 \pm 2300$ & $10500 \pm 4600$ \\
C-reactive protein (mg/dL)a & $0.04 \pm 0.1$ & $11.2 \pm 8.6$ \\
Albumin (g/dL)a & $4 \pm 0.2$ & $3 \pm 0.6$ \\
Phosphorus (mg/dL)a & $4.9 \pm 1.7$ & $3.9 \pm 1.4$ \\
Ferritin (ng/mL)b & $424 \pm 225$ & $597 \pm 452$ \\
iPTH (ng/L)b & $306 \pm 261$ & $215 \pm 185$ \\
LDL-C, (mg/dL)b & $112 \pm 34$ & $104 \pm 27$ \\
Uric acid (mg/dL)b & $5 \pm 1$ & $5 \pm 1$ \\
\hline
\end{tabular}

Mean \pm Standard deviations are shown, ${ }^{a} \mathrm{p}<0.05,{ }^{\mathrm{b}} \mathrm{p}>0.05$,

LDL-C: low density lipoprotein cholesterol, iPTH: intact parathyroid hormone.

peritonitis groups respectively, and the rest received continuous cycling peritoneal dialysis. The total time spent on PD was insignificantly longer in the control group than in the peritonitis group. The groups did not differ significantly with respect to staphylococcal nasal carriage, hypertension prevalence, transport status of peritoneum, occupations of patients, usage of proton pump inhibitors, antibiotics, immunosuppressive drugs, history of abdominal surgery, endoscopic procedure and constipation, mean data of all recorded nPCR, Kt/V calculations (Tab. 3).

Eight patients were transferred to hemodialysis in the peritonitis group. Among 31 episodes of CPD-related peritonitis, the main responsible organism was staphylacoccus aureus. The mean of all recorded laboratory parameters of groups is illustrated in the 
Table 4. The peritonitis group has found to have significantly less serum albumin, phosphorus levels and more C-reactive protein, white blood cell levels than the control group.

\section{Discussion}

Peritoneal dialysis is an accepted and widely used form of renal replacement therapy (6). Fortunately, peritonitis rates have been decreasing in the past years due to advances in PD techniques (like "Y" disconnect systems) (7). Yet peritonitis still remains the main complication involved in drop-out causes ${ }^{6}$. Most international authors have described gram-positive microorganisms as the major causative agents of peritonitis, likewise we have found staphylococcus aureus being the most frequent. Sociodemographical, nutritional factors, diabetes mellitus, PD modality, presence of tunnel or exit site infection have been reported as possible risk factors associated with the development of peritonitis $(8,9)$.

In this observation period of five years, our data have revealed that malnutrition, hygiene deficits, low education, low urine output, psychological problems and smoking may render the patient more prone to peritonitis in accordance with the reports of other authors. For instance, it was shown that peritonitis risk tended to decrease as education level increased $(9,10)$. Hypoalbuminemia is also very well known to be associated with a higher chance of developing peritonitis (9). As it comes to self hygiene neglect, poor sense of hygiene among patients was thought to be responsible for the high rate of peritonitis (11). It would be wise to make policy about home visits in detecting weakest point of PD practice to improve personal hygiene scores by insisting on strict hygienic measures. Smoking was accused to increase the risk of peritonitis as well and it was suggested to be considered in selecting peritoneal dialysis population (12). In this particular study, smoking history was found to render patients significantly more prone to peritonitis.

Reduced residual renal function was found to be related to both higher peritonitis risk and lower survival among CPD patients (13). The loss of residual renal function contributes to inflammation and malnutrition, which are both strong predictors of peritonitis and mortality. Our study revealed that urine output of more than 500 $\mathrm{mL} /$ day decreased peritonitis episodes. Thus our study supported that protective strategies to preserve residual renal functions might also reduce peritonitis rate.

Both hemodialysis and peritoneal dialysis patients have diminished quality of life scores compared to healthy controls. It was reported that low quality of life scores and depression are associated with higher comorbidity, poor nutritional status and lower residual renal functions (14). Likewise analysis of our data also revealed that patients with episodes of peritonitis had more psychological problems compared to those who had none. As suggested before, a rapid detection of psychosocial problems may enhance patient's management of illness and clinical outcome.

Many authors previously reported risk factors like anemia, dialysis modality, age of the patients, but these were not found to be significantly related to peritonitis development in this particular study. However, the present study had some limitations, such as being small, retrospective and single centred study. Therefore study should be carefully considered before results can be generalized.

In conclusion, lower rates of peritonitis lead to better preservation of the peritoneal membrane as well as higher survival and lower hospitalization rates. Hence it is worth emphasizing the importance of measures to ameliorate problems in nutritional, psychosocial, educational, self and environmental hygiene status among peritoneal dialysis patients to reach a better outcome.

\section{References}

1. Furkert JD, Zeier M, Schwenger V. Continuous ambulatory peritoneal dialysis- developments during changing times. Nephron Clin Pract 2008; 110: 139-143.

2. Charlson ME, Pompei P, Ales KL, MacKenzie CR. A new method of classifying prognostic comorbidity in longitudinal studies: development and validation. J Chronic Dis 1987; 40: 373-383.

3. Daugirdas JT, Depner TA, Grene T. Surface area normalized Kt/V a method of rescaling dialysis dose to body surface area implications for different size patients by gender. Semin Dial 2008; 21: 415-421.

4. Keshaviah PR, Nolph KD. Protein catabolic rate calculations in CAPD patients. ASAIO Trans 1991; 37: 400.

5. Twardowski ZJ. Clinical value of standardized equilibration tests in CAPD patients. Blood purif. 1989; 7: 95-108.

6. Krishnan M, Thodis E, Ikonomopoulos D, et al. Predictors of outcome following bacterial peritonitis in peritoneal dialysis. Perit Dial Int 2002; 22 (5): 573-581.

7. Stinghen AE, Baretti P, Pecoits-Filho R. Factors contributing to differences in peritonitis rates between centers and reagions. Perit Dial Int 2007; 27: 281-285.

8. Chow KM, Szeto CC, Leung CB, Law MC, Li PKT. Impact of social factors on patients on peritoneal dialysis. Nephrol Dial Transplant 2005; 20: 2504-2510.

9. Lobo JV, Villar KR, de Andrade Junior MP, Bastos Kde A. Predictor factors of peritoneal dialysis-related peritonitis. J Bras Nefrol 2010; 32: $156-164$.

10. Dogan S, Ekiz S, Yucel L, Ozturk S, Kazancioglu R. Relation of demographic, clinic and biochemical parameters to peritonitis in peritoneal dialysis. J Renal Care 2008; 34: 5-8.

11. Ozturk S, Yucel L, Guvenc S, ekiz S, Kazancioglu R. Assessing and training patients on peritoneal dialysis in their own homes can influence better practice. J Ren Care 2009; 35: 141-146.

12. Kotsanas D, Polkinghorne KR, Korman TM, Atkins RC, Brown F. Risk factors for peritoneal dialysis-related peritonitis: can we reduce the incidence and improve patient selection? Nephrology (Carlton) 2007; 12: 239-245.

13. Han SH, Lee SC, Ahn SV, et al. Reduced residual renal function is a risk of peritonitis in continuous ambulatory peritoneal dialysis patients. Nephrol dial transplant 2007; 22: 2653-2658.

14. Lew SQ, Piraino B. Quality of life and psychological issues in peritoneal dialysis patients. Semin Dial 2005; 18: 119-123.

Received August 14, 2011. Accepted November 30, 2011. 